\title{
TRASTORNO LÍMITE DE LA PERSONALIDAD: REVISIÓN SISTEMÁTICA DE LAS INTERVENCIONES TLP: Comparación de tratamientos
}

\author{
BORDERLINE PERSONALITY DISORDER: \\ SYSTEMATIC REVIEW OF INTERVENTIONS \\ TLP: Comparison of treatments
}

\author{
Eva Font Domènech \\ Universidad Nacional de Educación a Distancia (UNED). Facultad de Psicología. Madrid, España \\ ORCID: https://orcid.org/0000-0001-9116-1413 \\ Cómo referenciar este artículo/How to reference this article: \\ Font-Domènech, E. (2019). Trastorno Límite de la Personalidad: Revisión Sistemática de las Intervencio- \\ nes. Revista de Psicoterapia, 30(113), 197-212. https://doi.org/10.33898/rdp.v30i113.274
}

\section{Resumen}

El Trastorno Limite de la Personalidad (TLP) es un sindrome complejo y heterogéneo, caracterizado principalmente por la inestabilidad afectiva, interpersonal $y$ de la autoimagen. Durante los últimos años varios ensayos han valorado la eficacia de distintas estrategias psicoterapéuticas para tratar dicho trastorno. El objetivo del presente trabajo es ofrecer una revisión actualizada que permita analizar la evidencia procedente de los Ensayos Controlados Aleatorizados (ECA) mediante un abordaje cualitativo. La evidencia científica sugiere que las psicoterapias estructuradas contribuyen de manera significativa en la mejora de los cuadros limitrofes, tanto en su sintomatología como en su funcionamiento. Aunque se encuentran diferencias en los factores en que incide cada intervención, esto puede contribuir en la elección del tratamiento más adecuado, consiguiendo una visión dimensional y funcional del síntoma en un contexto más centrado en el paciente.

Palabras claves: Trastorno límite de la personalidad, Psicoterapias, Revisión

\begin{abstract}
Borderline Personality Disorder (BPD) is a complex and heterogeneous syndrome characterized primarily by affective, interpersonal, and self-image instability. During the last years several trials have evaluated the effectiveness of different psychotherapeutic strategies to treat this disorder. The objective of the present study is to provide an updated review to analyze the evidence from Randomized Controlled Trials (RCTs) through a qualitative approach. Scientific evidence suggests that structured psychotherapies contribute significantly to the improvement of borderline cases, both symptomatology and functioning. There are differences in the factors that affect each intervention, that can contribute to the choice of the most appropriate treatment, achieving a dimensional and functional vision of the symptom in a context patient centered health.
\end{abstract}

Keywords: Personality limitation disorder, Psychotherapies, Randomized controlled trial, Review

Fecha de recepción: 3/04/2019. Fecha de aceptación v1: 22/05/2019. Fecha de aceptación v2: 5/06/2019.

Correspondencia sobre este artículo:

E-mail: evafontd@hotmail.com

(C) 2019 Revista de Psicoterapia 


\section{Introducción}

En la Sección II del Manual Diagnóstico y Estadístico de los Trastorno Mentales (5, ${ }^{\mathrm{a}}$ Ed.: DSM-5; American Psychiatric Association [APA], 2013) encontramos que el TLP se caracteriza por la presencia de un patrón persistente de inestabilidad afectiva, interpersonal y de la autoimagen, con alteraciones de conducta marcadas por la impulsividad e ira inapropiada, con inicio en las primeras etapas de la edad adulta y que interfiere en varios contextos. Además, los sujetos con TLP pueden llegar a desarrollar ideas paranoides transitorias con el estrés 0 síntomas disociativos graves (APA, 2013; Zanarini y Jager-Hyman, 2009).

La heterogeneidad de los pacientes se hace patente al considerar la disparidad sintomatológica. Si tenemos en cuenta el diagnóstico categorial realizado con la sección II del DSM-5 (APA, 2013), encontramos que son necesarios cinco de los nueve criterios para realizar un diagnóstico de TLP (126 combinaciones posibles). Esto nos lleva a la posibilidad de encontrar dos sujetos que compartan diagnóstico con un sólo síntoma en común. Las presentaciones clínicas variables no sólo se presentan inter-sujeto, sino que también existen a nivel intra-sujeto de modo que pueden predominar unos rasgos y síntomas sobre otros dependiendo del momento vital del paciente (García, Martín y Otín, 2010).

Tanto el DSM-5 Sección II (2013) como el CIE-10 (OMS, 1992) son sistemas clasificatorios categoriales que presentan deficiencias: en ambos casos no se considera el grado de sintomatología del paciente. Además, debido a que los síndromes son entidades bien delimitadas, existe una alta tasa de comorbilidad y solapamiento entre diferentes categorías (Esbec y Echebúrua, 2014; González Robles et al., 2015; Gutiérrez y Vilar, 2014). No obstante, en la Sección III del DSM5 podemos observar un modelo híbrido (categórico dimensional) con el propósito de mitigar las limitaciones que presenta un modelo categorial puro.

La tasa de prevalencia del TLP se considera elevada, aunque no existe un acuerdo unánime. En el DSM-IV-TR (APA, 2000) se estima aproximadamente que el 2\% de la población general presenta TLP, mientras que entre la población atendida en los Servicios de Salud Mental Comunitarios se eleva hasta el 10\%. Sin embargo, otros autores como Kernberg y Mischels (2009) indican una tasa del 4\% entre la población general y del 20\% entre los usuarios de los Servicios de Salud Mental Comunitarios. Según la Guía de práctica clínica sobre el Trastorno Límite de la Personalidad (2011), el TLP es más frecuente en mujeres que en hombres con una relación estimada de 3:1. Los sujetos suelen ser diagnosticados en edades comprendidas entre los 19 y los 34 años y suelen pertenecer a grupos de nivel socioeconómico medio.

Los sujetos con TLP presentan una frecuencia de utilización de los servicios sanitarios más elevada que otros pacientes (Leichsenring, Cruse, New, Leweke, 2011), lo cual hace posible que exista una sobreestimación en las tasas de prevalencia (Cervera et al., 2005). En cuanto a la variabilidad sintomatológica, anteriormente mencionada, se relaciona con una elevada comorbilidad. Se estima 
que aproximadamente el $75 \%$ de los pacientes con TLP presentan otro trastorno mental, siendo los más comunes los trastornos depresivos, la ansiedad, el consumo de sustancias y los trastornos alimentarios (Barrachina et al., 2011). Por otra parte, alrededor del $80 \%$ de los sujetos con TLP cumplen con los criterios de otro trastorno de personalidad: se estima que entre el 30 y $38 \%$ presentan un Trastorno de Personalidad (TP) del Cluster A (principalmente Trastorno de Personalidad Paranoide); aproximadamente el $40 \%$ cumplen los criterios para otro de los TP del Cluster B (principalmente TP Antisocial seguidos por el TP Histriónico). Por último, también se ha estimado una elevada tasa de comorbilidad con trastornos del Cluster C, por ejemplo, en el TP por Evitación (del 20\%al 40\%) (Barrachina et al., 2011; Palomares et al., 2016).

El TLP se considera un trastorno grave por el elevado sufrimiento que ocasiona en los pacientes, la disfuncionalidad e incapacidad que ocasiona en diversas esferas de su vida (emocional, interpersonal y laboral) y el padecimiento de los sujetos que comparten el día a día con un TLP (familiares, pareja y amigos). Asimismo, nos encontramos con el único síndrome en el que figuran las conductas suicidas y parasuicidas como criterio diagnóstico. Se estima que la tasa de mortalidad en el TLP por suicidio es de 4-10\%, siendo este un elevado riesgo (Black et al., 2004; Zanarini Frankenburg, Hennen, Reich y Silk, 2005).

Como hemos indicado anteriormente, la heterogeneidad en la sintomatología, el solapamiento con otros cuadros clínicos y la elevada comorbilidad dificultan su diagnóstico, su diagnóstico diferencial y su delimitación. Sin embargo, este no es el único obstáculo que se presenta al tratar con pacientes con TLP, puesto que nos encontramos con un importante reto a la hora de establecer un tratamiento eficaz, ya que no solo debe hallarse una terapia que remita o disminuya la sintomatología, sino que esta debe contemplar las dificultades derivadas del funcionamiento limítrofe (alta tasa de abandonos, incumplimiento terapéutico, inestabilidad, pensamiento dicotómico, ira e impulsividad). Además, el funcionamiento de los sujetos con TLP, las autolesiones e intentos de suicidio ocasionan que estos pacientes sean usuarios habituales de los servicios médicos y de urgencias (Sansone y Sansone, 2015). Traduciéndose en un elevado coste institucional y una devaluación en su atención (Kjaer, Biskin, Vestergaard y Munk-Jorgensen, 2015; Romero-Cabello, González-Salas y Sánchez, 2016).

La gravedad del trastorno, la elevada prevalencia, la tasa de mortalidad, el coste Institucional, así como las dificultades que se presentan al aplicar una psicoterapia a pacientes con TLP, son factores que han propiciado la proliferación de estudios que evalúan la eficacia de tratamientos (Fernández-Guerrero y PalaciosVicario, 2017) y una mayor atención especializada para los pacientes con esta patología (García, Martín y Otín, 2010).

De hecho, en las últimas décadas se han utilizado varias psicoterapias para tratar el TLP. En la literatura encontramos estudios que evalúan la utilización de terapias de distintas orientaciones: psicodinámicas (Terapia basada en la 
Mentalización de Bateman y Fonagy; Terapia Focalizada en la Transferencia de Kernberg y Clarkin; Terapia interpersonal basada en el Modelo Conversacional de Hobson de Meares) o cognitivo-conductuales (Terapia Dialéctico Conductual de Linehan; Terapia Cognitivo-conductual para Trastornos de Personalidad de Davidson; Terapia de Esquemas de Young).

A continuación, se presenta una recopilación de los ensayos clínicos aleatorizados (ECA) disponibles que comparan intervenciones para el TLP, con el propósito de sintetizar la evidencia encontrada hasta el momento.

\section{Metodología}

\section{Estrategias de búsqueda}

Se ha realizado una búsqueda de la literatura publicada desde 1997 hasta 2017. Para ello, se han utilizado las bases de datos electrónicas PsycARTICLES, Psychology and Behavioral Sciencies Collection y PsycINFO, además del motor de búsqueda Linceo. El análisis bibliográfico se ha complementado con la consulta de manuales referenciados en los artículos y/o de interés por la temática tratada. Los descriptores de búsqueda para la identificación e inclusión de artículos han sido [Treatment] AND [Borderline Personality Disorder] AND [Radomized Trial] AND [Effectiveness], además, de figurar el término [Borderline] en el título.

\section{Criterios de selección}

Se ha integrado y analizado cualitativamente la información y los resultados de los estudios publicados cuyo objetivo principal era la valoración de la efectividad de diversas psicoterapias en el tratamiento del TLP. Para ello, se han incluido los ECA con muestra original y en que figurase la información de ella en el artículo, y que comparasen dos o más terapias. Se incluyeron aquellos ensayos en los que todos los participantes cumplieran los criterios del DSM-III-R (APA, 1987) y DSM-IVTR (APA, 2000) para el TLP y aceptaran participar en el estudio siendo asignados aleatoriamente a la condición experimental.

En cuanto a las características de los participantes, se descartaron los ensayos con individuos menores de 18 años, además se excluyeron también los ECA en los que los pacientes: a) presentaban cualquier trastorno psicótico (excepto episodios breves), b) formaban parte de la población presidaria, c) habían recibido la psicoterapia no habitual evaluada durante los seis meses anteriores al inicio del ensayo.

No han sido motivo de exclusión la presencia de trastornos por dependencia de sustancias, trastornos alimentarios y TP concurrentes, por la elevada comorbilidad presente en la práctica clínica. 


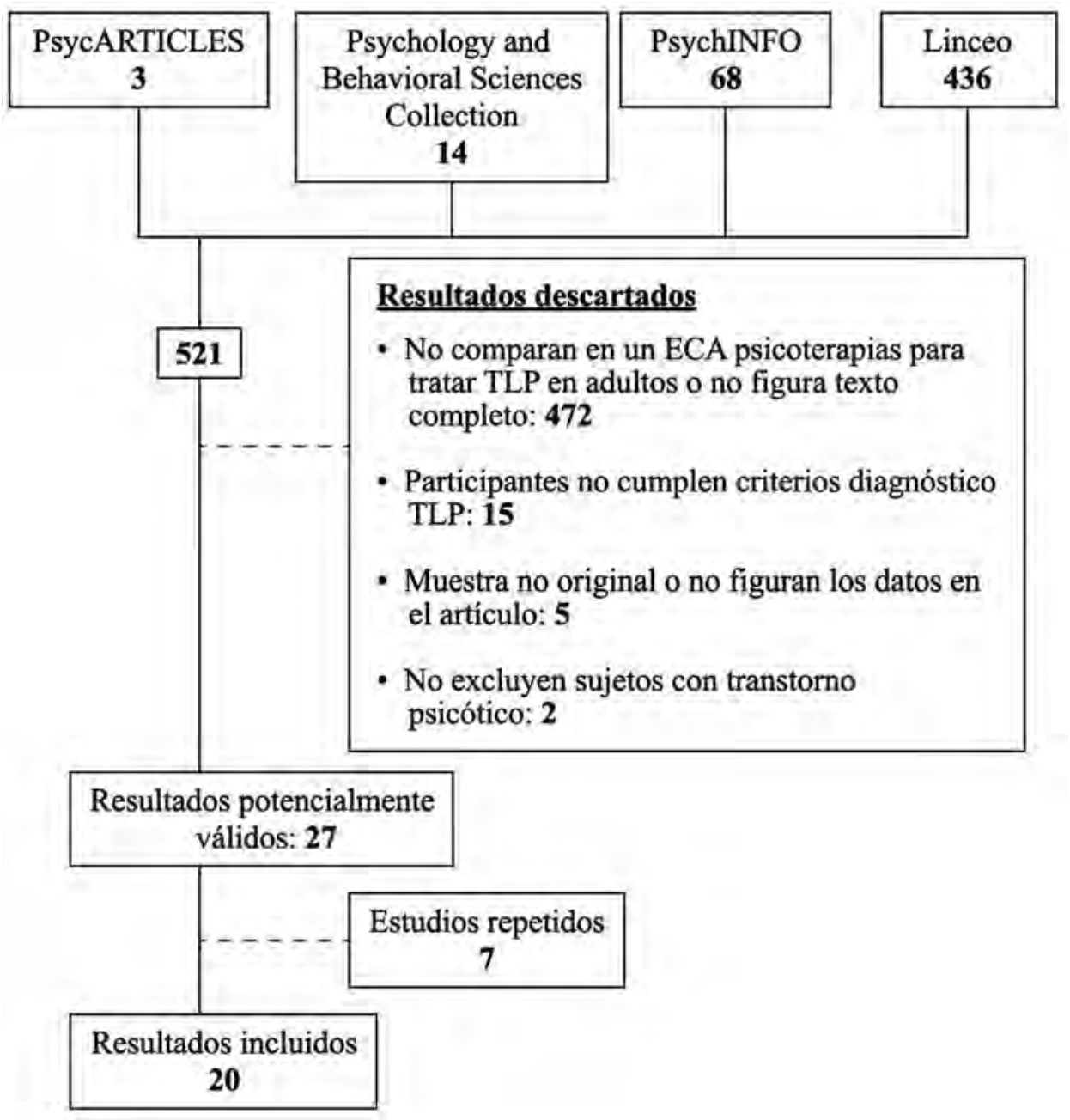

Figura 1. Diagrama de flujo de los estudios incluidos y exluidos para la revisión sistemática.

\section{Resultados}

A partir de la búsqueda en las bases de datos se obtuvieron un total de 521 artículos que fueron escrutados para su posible inclusión. Tras eliminar los duplicados y los que no cumplían los criterios de inclusión y exclusión, quedaron 21 ensayos (Figura 1) (Tabla 1).

\section{TDC}

En el año 1999, Linehan y colaboradores llevaron a cabo un ensayo clínico aleatorizado en el que se compararon Terapia Dialectico-Conductual (TDC) y el Tratamiento Habitual no estandarizado (TH), en el que reunió a 28 mujeres con y sin adicción (cumplían los criterios diagnósticos de trastorno por abuso de cocaína, 
opiáceos, anfetaminas, sedantes, hipnóticos, benzodiacepinas o policonsumo del DSM-III-R) y las trataron durante 12 meses. En este estudio se valoró la conducta de consumo, en cinco ocasiones, mediante análisis de orina (antes de empezar el tratamiento, a los 4,8 y 12 meses, y finalmente se realizó una valoración posttratamiento a los 16 meses). Entre los resultados obtenidos se observó que el efecto de TDC fue significativamente superior en la disminución de consumo de drogas, en la adherencia al tratamiento y en el ajuste social y global. Tanto en TDC como en TH no se evidenciaron cambios en los sentimientos de ira, ni en las conductas para-suicidas.

Posteriormente Turner (2000) estudió el efecto de las mismas terapias, en este estudio la muestra estaba compuesta por 24 sujetos (varones y mujeres) con TLP que también fueron tratados durante 12 meses con TDC y TH (en este caso la terapia estándar se centraba en cada caso). En este ensayo, en cambio, se encontró que con TDC se producía una reducción significativa en las conductas suicidas y autolesivas, en la ideación suicida, y en los sentimientos de ira. Además, se produjeron cambios significativos favorables en la frecuencia de los días de hospitalización, dominio emocional y funcionamiento de la salud mental global en ambas condiciones terapéuticas, pero no se observaron cambios significativos en ansiedad, enojo e impulsividad.

Koons et al. (2001) realizaron un ensayo aleatorizado con 20 mujeres y nuevamente compararon la TDC con TH después de 6 meses de tratamiento. Se obtuvo una mejora significativa en ambos grupos en ansiedad y en sintomatología depresiva, que fue estadísticamente superior en TDC. Del mismo modo, se observó una reducción en el número de criterios para TLP. La TDC también mostró cambios relevantes en la disminución de conductas para-suicidas, ideación suicida, ira y presencia de disociación. En este trabajo no se encontraron diferencias significativas intra e intergrupal en el número de admisiones psiquiátricas.

Verheul et al. (2003) desarrollaron un ensayo con 58 mujeres, las pacientes recibieron TDC o TH durante 12 meses. Observaron que las conductas autolesivas y la impulsividad experimentaban una disminución significativamente superior en el grupo TDC. Los intentos de suicidio, sintomatología característica del TLP y problemas de adicción, aunque disminuyeron, no mostraron cambios significativos.

Linehan y colaboradores realizaron otro ensayo publicado en 2006, en este caso se disponía de una muestra de 101 mujeres que recibieron tratamiento durante 12 meses. Se comparó TDC y Tratamiento Comunitario, en los que encontraron mejoras en todas las variables consideradas, las cuales incluían hospitalizaciones en el área de psiquiatría, intentos autolíticos, ideación suicida y sintomatología depresiva. Sin embargo, no se encontraron diferencias significativas entre ambas condiciones experimentales.

En 2009 un equipo de investigación encabezado por McMain llevó a cabo otro ensayo clínico aleatorizado para comparar TDC y TH. Se detectaron cambios favorables en ambos grupos en la mayoría de variables como conductas autolesivas, 
duración de las hospitalizaciones en psiquiatría, sintomatología característica del TLP y depresiva, angustia y en funcionamiento interpersonal. La mejoría fue evidente en ambos grupos, pero sin diferencia significativa. No se observaron cambios en la calidad de vida de los sujetos.

En el mismo año se publicó otro ECA (Soler et al., 2009) que compraba los resultados después de tres meses de tratamiento (TDC vs. TH). TDC resultó significativamente superior en la disminución la de sintomatología depresiva y ansiosa, además se observó una reducción en la sintomatología psiquiátrica relevante. Aunque, también encontraron una tendencia a la mejora en la expresión de ira y hostilidad, estos cambios no fueron significativos. Con la aplicación de la TDC se obtuvo una disminución significativa en la inestabilidad afectiva e interpersonal, con $\mathrm{TH}$ la tendencia fue favorable pero no significativa. La impulsividad se redujo en ambos grupos, pero solo en el grupo TDC fue relevante. La sensación de vacío, aunque disminuyó, los cambios no fueron significativos.

La TDC fue comparada nuevamente con TH (Carter, Willcox, Lewin, Conrad y Bendit, 2010), cuya muestra estaba formada por 73 mujeres y el tratamiento tuvo una duración de 6 meses. Encontraron un efecto significativamente beneficioso en tres de los cuatro ámbitos de calidad de vida (físico, psicológico y ambiental) a favor de la TDC. Sin embargo, en el número de hospitalizaciones y la ejecución de conductas autolesivas deliberadas no encontraron mejoras significativas.

En 2012, Bedics, Atkins, Comtois y Linehan realizaron un ECA con una muestra de 101 mujeres que fueron asignadas al azar para recibir TDC o TH. En el que ambas intervenciones fueron aplicadas durante 12 meses. En los resultados obtuvieron que los pacientes que recibieron TDC presentaban una afiliación terapéutica significativamente superior que con TH. Además, también encontraron una disminución relevante en las conductas autolesivas y una mayor autoafirmación de los introyectos en los sujetos con TDC.

En un ensayo se combinó TDC con TH y se comparó con TH (Kramer et al., 2016). Los autores encontraron una tendencia favorable en ambas condiciones experimentales en disminución de angustia, mejora en funcionamiento interpersonal y en ajuste social. Observaron que estos cambios fueron significativamente superiores en el grupo TDC y TH. No encontraron cambios significativos en el rechazo a la ira en ninguno de los grupos. En este ensayo, también valoraron la gestión de la ira con asertividad y el rechazo a la hostilidad. Sin embargo, no se hallaron cambios significativos en los resultados globales.

\section{TCE y PE}

Farrell, Shaw y Webber en el 2009, realizaron un estudio para valorar la efectividad del TH cuando se le añaden 30 sesiones de Terapia Centrada en los Esquemas (TCE). Para ello, utilizaron una muestra de 32 mujeres que se asignaron a TH o TH y TCE durante 8 meses. Así encontraron, que el grupo con TCE mejoraba significativamente en las variables sintomatología TLP y funcionamiento global. 
Sin embargo, en la severidad sintomatológica no encontraron cambios en el grupo TH, y en el grupo TCE, aunque disminuyó, no fue significativo.

La TCE se combinó con la Psicoeducación (PE) para comparar la eficacia con el TH (Leppänem, Hakko, Sintonen y Lindeman, 2015). En este ensayo ambas condiciones experimentales resultaron ser efectivas en la reducción de la sintomatología limítrofe, en la inestabilidad interpersonal, en la perturbación de la identidad, en la sensación de vacío y en la ira. El grupo que fue tratado con TCE y PE, además, hallaron una reducción significativa en impulsividad, conductas parasuicidas, ideación paranoide y disociativa. Tabla 1. Síntesis de resultados de los ensayos incluidos en la revisión.

Aunque la tendencia fue favorable, no se obtuvieron mejoras relevantes en ningún grupo en temor al abandono e inestabilidad afectiva.

Pascual y colaboradores en el 2015, contrastaron los resultados obtenidos con la aplicación de una intervención basada en la Psicoeducación (PE) y otra en la Rehabilitación Cognitiva (RC) con una duración de 4 meses. La sintomatología característica del TLP no mostró cambios significativos, lo mismo encontraron en impulsividad. Los sujetos mostraron una reducción relevante en ansiedad en ambos grupos. La PE resultó superior en la disminución de la sintomatología depresiva, y por otro lado, la RC mostró una mejoría superior en el funcionamiento global de los pacientes.

\section{STEPPS}

Blum y colaboradores en el 2008, realizaron un ensayo con 134 sujetos, aplicando durante 5 meses el TH a un grupo de pacientes y TH combinado con "Systems training for emotional predictability and problem solving" (STEPPS) en el otro. Encontraron una disminución de la sintomatología TLP significativamente superior en el grupo que recibió STEPPS. En afectividad negativa, ajuste social, intentos de suicidio y autolesiones observaron una tendencia favorable, sin embargo, las diferencias no fueron significativas.

\section{TDC y PFT}

Clarkin, Levy, Lenzenweger y Kernberg en el 2007, realizaron un estudio en el que evaluaban tres tratamientos distintos. Las tres intervenciones mostraron beneficios significativos en: la disminución de sintomatología psiquiátrica, depresiva y ansiosa, también obtuvieron mejoras relevantes en el funcionamiento global $\mathrm{y}$ ajuste social. La ideación y comportamientos suicidas disminuyeron significativamente con la aplicación de TDC y Psicoterapia Focalizada en la Transferencia (PFT). Por otra parte, observaron la reducción significativa de presencia de irritabilidad agresión verbal y heteroagresión solo en el grupo PFT. La TDC fue la única terapia con la que no obtuvieron una disminución significativa de la ira. 


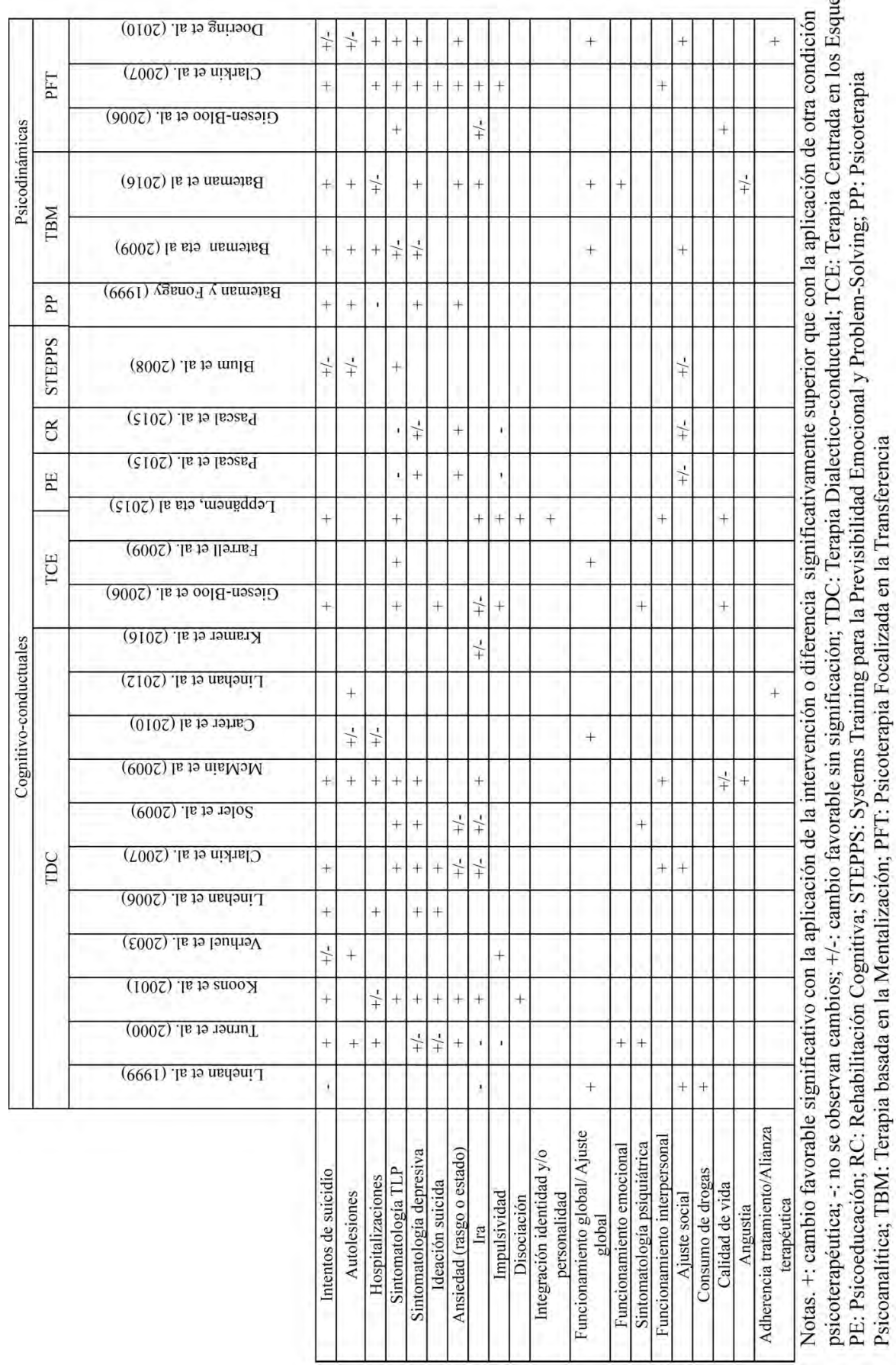

Tabla 1. Síntesis de resultados de los ensayos incluidos en la revisión. 


\section{PP}

Bateman y Fonagy en 1999, reunieron 38 pacientes con TLP para la realización de un ECA que comparase Psicoterapia Psicoanalítica (PP) y TH. Con PP obtuvieron mejoras significativas en cuatro de las variables estudiadas (conductas autolesivas y autolíticas, sintomatología depresiva y ansiedad). La duración de las hospitalizaciones se mantuvo en el grupo PP, mientras que con el TH aumentaron durante los últimos meses de tratamiento. En el grupo PP se encontró una disminución significativa en la gravedad sintomatológica, pero no en la disminución de los síntomas. La mejora en el ajuste social y el funcionamiento interpersonal fue significativamente superior en los sujetos que recibieron PP.

\section{TBM}

Bateman y Fonagy (2009) utilizaron una muestra de 134 sujetos para comparar la efectividad de Terapia basada en la Mentalización (TBM) y TH después de aplicar el tratamiento durante 18 meses. Se observaron mejoras relevantes en ambas condiciones, en todas las variables de resultado las variables de resultado como intentos de suicidio, presencia de autolesiones, número de hospitalizaciones, funcionamiento global, sintomatología depresiva y psiquiátrica, y ajuste social. Los pacientes asignados al azar a TBM mostraron una disminución más pronunciada de los problemas autoinformados y clínicamente significativos, incluidos los intentos de suicidio y la hospitalización.

En 2016, Bateman, O’Connell, Lorenzini, Gardner y Fonagy realizaron un ensayo en el que se comparaba la TBM con TH en pacientes con TLP y Trastorno antisocial comórbido después de 18 meses de tratamiento. La muestra estaba compuesta por 40 sujetos que presentaban algún episodio de intento de suicidio y/ o autolesiones en los últimos 6 meses. En los resultados encontraron una disminución significativamente superior con TBM en: sintomatología paranoica, intentos de suicidio (teniendo en cuenta los efectos mixtos de la regresión de Poisson), autolesiones e intentos de autolesión, sintomatología depresiva, ansiedad, y hostilidad. Aunque en ambos grupos se presentase una mejoría, las diferencias no fueron relevantes en: ira, cantidad de ingresos hospitalarios y de estrés. En ambas condiciones los pacientes mostraron mejora en el funcionamiento global e interpersonal, aunque en el grupo TBM fueron significativamente superiores.

\section{PTF}

Doering y colaboradores en el 2010 contrastaron la efectividad de la PTF y TH, el tratamiento fue aplicado durante 12 meses a una muestra compuesta únicamente por mujeres. Mientras que en el grupo TH los sujetos no mostraron cambios significativos en la proporción de pacientes que presentaron conductas parasuicidas ni en el número de hospitalizaciones. En ambos resultados PTF fue significativamente superior. La sintomatología característica del TLP experimentó una disminución significativa en ambos grupos. La PTF mostró resultados superiores en el funcio- 
namiento global y la organización de la personalidad. En ambas condiciones encontraron tendencias favorables en sintomatología depresiva, ansiedad y psicopatología general. Sin embargo, ninguna de las dos condiciones ocasionó mejoras significativas en las conductas autolesivas.

\section{PTF y TCE}

En un ensayo se comparó el tratamiento durante 36 meses de TCE y PTF (Giesen-Bloo et al., 2006). Aunque ambas terapias mostraron beneficios la TCE fue superior en todas las variables (calidad de vida, sintomatología TLP, temor al abandono, impulsividad, conductas autolíticas, e ideación suicida y paranoica) exceptuando la ira en que no se hallaron cambios relevantes.

\section{Discusión}

Los casos de TLP suponen un gran desafío en la práctica clínica por su difícil tratamiento, complejidad y gravedad. En la revisión que se ha presentado se han tenido en cuenta los ECA publicados durante los últimos 20 años para valorar la eficacia que muestran los distintos tratamientos. Como se puede observar, encontramos una gran variedad de psicoterapias utilizadas para el TLP y, en términos generales, se podría afirmar que la mayoría de los tratamientos recogidos en esta revisión producen mejoras en los cuadros limítrofes, independientemente de cuales sean las técnicas utilizadas o las teorías sobre las que se sustentan.

La sintomatología característica del TLP disminuye con la aplicación de cualquiera de los tratamientos considerados: TDC (Koons et al., 2001; McMain, et al., 2009; Soler, et al., 2009; Verheul et al., 2003), PFT (Doering et al., 2010; GiesenBloo, 2006), TCE (Farrell et al., 2009; Giesen-Bloo, 2006), STEPPS (Blum et al., 2008), y, TCE y PE (Leppänem et al., 2015). Sin embargo, con la aplicación de PE o RC (Pascal et al., 2015) no se encontraron cambios. Esto podría significar que la aplicación de estos componentes, por sí solos, no son suficientes para la reducción de la sintomatología limítrofe.

Las conductas parasuicidas se han utilizado como variable resultado en la mayoría de los ensayos, mostrando una tendencia significativa en la disminución de dichas conductas en la mayoría de ellos, con la aplicación de la TDC (Clarkin et al., 2007; Koons et al., 2001; Linehan et al., 2006; McMain et al., 2009; Turner, 2000; Verheul et al., 2003), la TBM (Bateman y Fonagy, 2009; Bateman, et al., 2016), la PFT (Clarkin et al., 2007; Doering et al., 2010; Giesen-Bloo et al., 2006), la TCE (Giesen-Bloo et al., 2006), del programa STEPPS (Blum et al., 2008), la PP (Bateman y Fonagy, 1999), y también con la TCE y PE (Leppänem et al., 2015), independientemente de si el tratamiento era de enfoque cognitivo- conductual o psicodinámico. Exceptuando el publicado por Linehan et al. (1999), en dónde no se encuentra dicha tendencia con la TDC. Entre los posibles motivos de las diferencias halladas, podría considerarse la presencia de trastorno por dependencia presente en todos los participantes de este ensayo y la alta tasa de abandono. 
Los pacientes con TLP son usuarios recurrentes en los centros sanitarios debido a su sintomatología y funcionamiento, la mejora en el trastorno puede traducirse indirectamente como un descenso en las admisiones e ingresos hospitalarios. Esta variable ha sido considerada en varios ensayos, con la TDC se obtiene una disminución significativa (McMain et al., 2009; Turner, 2000; Verheul et al., 2003) y una tendencia a la reducción no significativa (Carter et al., 2010; Koons et al., 2001). Con la TBM los resultados muestran una reducción significativa (Bateman y Fonagy, 2009; Bateman et al., 2016), lo mismo sucede con la aplicación de PFT (Giesen-Bloo et al., 2006; Clarkin, et al., 2007). Sin embargo, con la PP no se encontraron cambios relevantes (Bateman y Fonagy, 1999).

Con las psicoterapias no habituales (TDC, TBM, PFT, TCE, STEPPS, PP, PE y RC) se observa una tendencia general en la disminución de conductas autolesivas, sintomatología depresiva, ideación suicida y en ansiedad (Bateman y Fonagy, 1999, 2009; Bateman et al., 2016; Bedics, et al., 2012; Blum, et al., 2008; Carter, et al., 2010; Clarkin, et al., 2007; Doering et al., 2010; Giesen-Bloo et al., 2006; Koons et al., 2001; Linehan et al., 2006; McMain et al., 2009; Pascual et al., 2015; Soler et al., 2009; Turner, 2000; Verheul et al., 2003;).

El funcionamiento global de los pacientes mejora significativamente en todos los ensayos en los que se ha considerado esta variable, con la TDC (Carter et al., 2010; Linehan, et al., 1999;), la TBM (Bateman y Fonagy, 2009; Bateman et al., 2016), la PFT (Doering et al., 2010) y la TCE (Farrell et al., 2009). También se hallaron los mismos resultados con la RC (Pascual et al., 2015). Sin embargo, con la aplicación de PE no se encontraron cambios significativos (Pascual et al., 2015). Asimismo, esta tendencia de mejora también se muestra con el funcionamiento interpersonal, ajuste social y calidad de vida, aunque no siempre significativa (Bateman y Fonagy, 2009; Blum et al., 2008; Doering et al., 2010; Clarkin et al., 2007; Giesen-Bloo et al., 2006; Kramer et al., 2016; Leppänem, et al., 2015; Linehan et al., 1999; McMain et al., 2009).

La angustia emocional con la que conviven los sujetos con TLP les ocasiona un malestar que suele desembocar en comportamientos autolesivos y conductas de riesgo. La disminución de la angustia supone una mejora en la calidad de vida objetiva y subjetiva de los pacientes. Se ha observado que con la TDC puede reducirse significativamente la angustia (McMain, et al., 2009; Kramer, et al., 2016), y con la utilización de la TBM se ha encontrado una tendencia favorable, pero no significativa (Bateman et al., 2016). Entre las conductas de riesgo más habituales en los pacientes con TLP encontramos el consumo de tóxicos. La TDC ha mostrado que puede ser efectiva para la reducción de patrones de consumo (Linehan et al., 1999; Verheul et al., 2003).

Respecto a la disminución de la ira (Bateman et al., 2016; Clarkin et al., 2007; Giesen-Bloo et al., 2006; Koons et al., 2001; Kramer et al., 2016; Leppänem et al., 2015; Linehan et al., 1999; McMain et al., 2009; Soler et al., 2009; Turner, 2000) y la impulsividad (Clarkin et al., 2007; Giesen-Bloo et al., 2006; Leppänem et al., 
2015; Pascual et al., 2015; Soler et al.; 2009; Turner, 2000; Verheul et al., 2003), encontramos una gran heterogeneidad en los resultados. La utilización de distintos instrumentos y subescalas para la valoración de ambas variables puede ocasionar la disparidad en los resultados obtenidos con la aplicación de las psicoterapias. La variable resultado ira, vemos que ha sido considerada en diez ensayos y para su medición se han utilizado ocho instrumentos distintos. El análisis del efecto de las psicoterapias en estos dos componentes limítrofes presenta gran dificultad por la diversidad en los efectos hallados y los instrumentos usados.

Kernberg (1987) propone un diagnóstico estructural en el que considera la ausencia o baja integración de la identidad como una de las tres características fundamentales de las personalidades limítrofes. Considerándose un atributo de esta patología (DSM-5, 2013; Kernberg, 1987), sorprende que solo figure en dos de los ensayos como factor para valorar el efecto de las intervenciones. Los resultados muestran que con la PFT (Doering et al., 2010) y la TCE y PE (Leppänem et al., 2015) se obtuvieron mejoras notables en la integración de la identidad de los pacientes con TLP.

Las alteraciones de la identidad se relacionan con los estados disociativos en los sujetos con TLP (Mosquera y González, 2011), en los que comúnmente existe un distanciamiento entre la percepción subjetiva del paciente y la experiencia física y emocional. La reducción de las ideaciones disociativas fue significativa con la aplicación de la TDC (Koons et al. 2001) y de la TCE y PE (Leppänem et al., 2015).

En los ensayos se ha encontrado una disminución importante de sintomatología psiquiátrica global aplicando la TDC (Clarkin et al., 2007; Soler et al., 2009; Turner, 2000), la PFT (Clarkin et al., 2007) y la TCE (Farrell et al., 2009). Aunque la tendencia fue favorable, los resultados no fueron significativos con la aplicación de TBM (Bateman y Fonagy, 2009) y PP (Bateman y Fonagy, 1999).

La alianza terapéutica se considera una condición necesaria para que el tratamiento pueda ser efectivo (Semerari y Dimaggio, 2008). Con la aplicación de TDC y PFT (Bedics et al., 2012; Doering, et al., 2010; Linehan et al., 1999) se consiguió crear un vínculo terapeuta-paciente sólido. Con las otras terapias no existe evidencia que valore esta variable.

Al considerar las tasas de deserción hallamos una gran heterogeneidad: TDC del 4\%- 47\%, TBM del 27\%, PFT del 23\% - 51\%, TCE del 0\%- 27\%, STEPPS del $31 \%$, PP del $12 \%$, TCE y PE del $17 \%$, PE del $2 \%$ y RC del $28 \%$. Es necesario tener en cuenta que los criterios para considerar que un paciente abandona la terapia son distintos en los diversos estudios. Además, sería conveniente valorar si las diferencias encontradas son consecuencia de los beneficios o carencias de las terapias y/ o de las habilidades de los terapeutas. El análisis independiente de los abandonos permitiría evaluar si la terapia específica mejora la adherencia.

Esta revisión no pretende establecer una jerarquización de los tratamientos según su eficacia. Según las directrices de la American Psychiatric Association (APA, 2001) se prioriza la valoración de los riesgos para la integridad del paciente 
y la gestión flexible del caso para la elección del tratamiento del TLP. Con el análisis de los resultados obtenidos en los ensayos se han podido identificar algunos de los factores en que incide cada terapia, haciendo posible la elección de la intervención según las variables en que se pretende incidir. Por otra parte, se considera la valoración de los distintos componentes de los tratamientos en futuras investigaciones para posibilitar la integración y combinación de los elementos de forma que las intervenciones puedan centrarse en cada caso y se sustenten sobre una base empírica. Así pues, se aboga por un enfoque ecléctico centrado en el paciente para el tratamiento del TLP.

Finalmente, como limitaciones de esta revisión hay que remarcar que existe una cierta falta de homogeneidad metodológica en los estudios analizados a nivel de instrumentos de evaluación, formación de los psicoterapeutas y duración de las intervenciones. La comparación de la eficacia entre las distintas terapias se ve dificultada por la utilización de distintas pruebas para medir los mismos constructos. Además, encontramos diferencias significativas entre la duración de las intervenciones (3- 36 meses). Asimismo, se considera una limitación la utilización de ensayos con un tamaño muestral inferior a 15 sujetos en cada grupo por el sesgo potencial que supone.

Aunque se ha encontrado que los tratamientos presentados muestran cierta eficacia en el tratamiento del TLP, sería de interés que se considerase la homogeneización de los instrumentos y variables de los ensayos en futuras investigaciones para facilitar las comparaciones entre las intervenciones.

\section{Referencias bibliográficas}

American Psychiatric Association (1987). Diagnostic and Statistical Manual of Mental Disorders ( $3^{\text {th }}$ Ed., text Rev.). Washington, DC: American Psychiatric Publishing.

American Psychiatric Association (2000). Diagnostic and Statistical Manual of Mental Disorders (4 ${ }^{\text {th }}$ Ed., text Rev.). Washington, DC: American Psychiatric Publishing.

American Psychiatric Association (2001). Practice guideline for the treatment of patients with borderline personality disorders, vol.110. Washington, DC: American Psychiatric Publishing.

American Psychiatric Association (2013). Diagnostic and Statistical Manual of Mental Disorders ( $5^{\text {th }}$ Ed., text Rev.). Washington, DC: American Psychiatric Publishing.

Barrachina, J., Pascual, J. C., Ferrer, M., Soler, J., Rufat, M. J., Andión, O., Tiana, T., Martín-Blanco, A., Casas, M. y Pérez, V. (2011). Axis II comorbidity in borderline personality disorder is influenced by sex, age, and clinicalseverity. Comprehensive Psychiatry, 52(6), 725-730. https://bit.ly/2RVbmpJ

Bateman, A. y Fonagy, P. (1999). Effectiveness of partial hospitalization in the treatment of borderline personality disorder: a randomized controlled trial. American Journal of Psychiatry, 156(10), 1563-1569. https:// doi.org/10.1176/ajp.156.10.1563

Bateman, A. y Fonagy, P. (2009). Randomized controlled trial of outpatient mentalization-based treatment versus structured clinical management for borderline personality disorder. American Journal of Psychiatry, 166(12), 1355-1364. https://doi.org/10.1176/appi.ajp.2009.09040539

Bateman, A., O'Connell, J., Lorenzini, N., Gardner, T. y Fonagy, P. (2016). A randomised controlled trial of mentalization-based treatment versus structured clinical management for patients with comorbid borderline personality disorder and antisocial personality disorder. BMC psychiatry, 16(1), 304. https:// doi.org/10.1186/s12888-016-1000-9 
Bedics, J. D., Atkins, D. C., Comtois, K. A. y Linehan, M. M. (2012). Treatment differences in the therapeutic relationship and introject during a 2-year randomized controlled trial of dialectical behavior therapy versus nonbehavioral psychotherapy experts for borderline personality disorder. Journal of Consulting and Clinical Psychology, 80(1), 66. https://doi.org/10.1037/a0026113

Black, D. W., Blum, N., Pfohl, B. y Hale, N. (2004). Suicidal behavior in borderline personality disorder: prevalence, risk factors, prediction, and prevention. Journal of personality disorders, 18(3), 226-239. https://doi.org/10.1521/pedi.18.3.226.35445

Blum, N., St. John, D., Pfohl, B., Stuart, S., McCormick, B., Allen, J., ...y Black, D. W. (2008). Systems Training for Emotional Predictability and Problem Solving (STEPPS) for outpatients with borderline personality disorder: a randomized controlled trial and 1-year follow-up. American Journal of Psychiatry, 165(4), 468478. https://doi.org/10.1176/appi.ajp.2007.07071079

Carter, G. L., Willcox, C. H., Lewin, T. J., Conrad, A. M. y Bendit, N. (2010). Hunter DBT project: randomized controlled trial of dialectical behaviour therapy in women with borderline personality disorder. Australian \& New Zealand Journal of Psychiatry, 44(2), 162-173. https://doi.org/10.3109\%2F00048670903393621

Clarkin, J. F., Levy, K. N., Lenzenweger, M. F. y Kernberg, O. F. (2007). Evaluating three treatments for borderline personality disorder: a multiwave study. American Journal of Psychiatry, 164(6), 922-928. https:// doi.org/10.1176/ajp.2007.164.6.922

Doering, S., Hörz, S., Rentrop, M., Fischer-Kern, M., Schuster, P., Benecke, C., ... y Buchheim, P. (2010). Transference-focused psychotherapy v. treatment by community psychotherapists for borderline personality disorder: randomised controlled trial. The British Journal of Psychiatry, 196(5), 389-395. https:// doi.org/10.1192/bjp.bp.109.070177

Esbec, E. y Echeburúa, E. (2014). La evaluación de los trastornos de la personalidad según el DSM-5: recursos y limitaciones. Terapia Psicológica, 32(3), 255-264. Recuperado de: https://bit.ly/2XVB5nu

Farrell, J. M., Shaw, I. A. y Webber, M. A. (2009). A schema-focused approach to group psychotherapy for outpatients with borderline personality disorder: a randomized controlled trial. Journal of Behavior Therapy and Experimental Psychiatry, 40(2), 317-328. https://doi.org/10.1016/j.jbtep.2009.01.002

Fernández-Guerrero, M. J. y Palacios-Vicario, B. (2017). El trastorno límite de personalidad en la producción científica publicada en revistas editadas en España. Clínica y Salud, 28(3), 147-153. https://bit.ly/32bYFLK

García, M., Martín, M. y Otín, R. (2010). Tratamiento integral del trastorno límite de personalidad. Revista de la Asociación Española de Neuropsiquiatría, 30(2), 263-278. Recuperado de: https://bit.ly/2XubWMa

Giesen-Bloo, J., Van Dyck, R., Spinhoven, P., Van Tilburg, W., Dirksen, C., Van Asselt, T., ... y Arntz, A. (2006). Outpatient psychotherapy for borderline personality disorder: randomized trial of schema-focused therapy vs transference-focused psychotherapy. Archives of general psychiatry, 63(6), 649-658. https:// doi.org/10.10017archpsyc.63.6.649

González Robles, A., García Palacios, A., Molinari, G. y Del Río González, E. (2015). DSM-5: avances en la clasificación y el diagnóstico de los trastornos mentales. Ágora de Salud, 1, 115-126. http:// dx.doi.org/10.6035/AgoraSalut.2015.1.8

Grupo de trabajo de la guía de práctica clínica sobre trastorno límite de la personalidad. Fórum de Salud Mental y AIAQS (2011). Guía de práctica clínica sobre trastorno límite de la personalidad. Barcelona: Agènciad'Informació, Avaluació i Qualitat en Salut.

Gutiérrez, F. y Vilar, A. (2014). Trastornos de la personalidad en el DSM-5. Cuadernos de Medicina Psicosomática y Psiquiatría, 110, 49-52.

Kernberg, O. F. y Mischels, R. (2009). Borderline personality disorder. American Journal of Psychiatry, 166, 505508. https://doi.org/10.1176/appi.ajp.2009.09020263

Kjaer, J. N., Biskin, R., Vestergaard, C. H. y Munk-Jørgensen, P. (2015). A nationwide study of mortality in patients with borderline personality disorder. European Psychiatry, 30, 202. https://bit.ly/2xz2oVz

Koons, C. R., Robins, C. J., Tweed, J. L., Lynch, T. R., Gonzalez, A. M., Morse, J. Q., ... y Bastian, L. A. (2001). Efficacy of dialectical behavior therapy in women veterans with borderline personality disorder. Behavior therapy, 32(2), 371-390. https://bit.ly/32e7uVg

Kramer, U., Pascual Leone, A., Berthoud, L., Roten, Y., Marquet, P., Kolly, S., ... y Page, D. (2016). Assertive anger mediates effects of dialectical behaviour informed skills training for borderline personality disorder: A randomized controlled trial. Clinical psychology \& psychotherapy, 23(3), 189-202. https://bit.ly/32apDDE

Leichsenring, F., Leibing, E., Cruse, J., New, A. S. y Leweke F. (2011). Borderline personality disorder. Lancet, 377, 74-84. https://bit.ly/2L4mOP0 
Leppänen, V., Hakko, H., Sintonen, H. y Lindeman, S. (2016). Comparing effectiveness of treatments for borderline personality disorder in communal mental health care: The Oulu BPD Study. Community mental health journal, 52(2), 216-227. https://doi.org/10.1007/s10597-015-9866-4

Linehan, M. M., Schmidt, H., Dimeff, L. A., Craft, J. C., Kanter, J. y Comtois, K. A. (1999). Dialectical behavior therapy for patients with borderline personality disorder and drug dependence. The American journal on addictions, 8(4), 279-292. https://doi.org/10.1080/105504999305686

Linehan, M. M., Comtois, K. A., Murray, A. M., Brown, M. Z., Gallop, R. J., Heard, H. L., ... y Lindenboim, N. (2006). Two-year randomized controlled trial and follow-up of dialectical behavior therapy vs therapy by experts for suicidal behaviors and borderline personality disorder. Archives of general psychiatry, 63(7), 757-766. https://doi.org/10.1001/archpsyc.63.7.757

McMain, S. F., Links, P. S., Gnam, W. H., Guimond, T., Cardish, R. J., Korman, L. y Streiner, D. L. (2009). A randomized trial of dialectical behavior therapy versus general psychiatric management for borderline personality disorder. American Journal of Psychiatry, 166(12), 1365-1374. https:// doi.org/10.1176/appi.ajp.2009.09010039

Mosquera, D. y González, A. (2011). Del apego temprano a los síntomas del trastorno límite de personalidad. Revista Digital de Medicina Psicosomática y Psicoterapia. Recuperado en: https://bit.ly/30hlKuN

Organización Mundial de la Salud (OMS) (1992). Décima revisión de la clasificación internacional de las enfermedades. Trastornos mentales y del comportamiento. Descripción clínicas y pautas para el diagnóstico, Madrid, España: Méditor.

Palomares, N., McMaster, A., Díaz-Marsá, M., de la Vega, I., Montes, A. y Carrasco, J. L. (2016). Comorbilidad con el eje II y funcionalidad en pacientes graves con trastorno límite de la personalidad. Actas Españolas de Psiquiatría, 44(6), 212-21.

Pascual, J. C., Palomares, N., Ibáñez, Á., Portella, M. J., Arza, R., Reyes, R., ... y Carrasco, J. L. (2015). Efficacy of cognitive rehabilitation on psychosocial functioning in Borderline Personality Disorder: a randomized controlled trial. BMC psychiatry, 15(1), 255. https://doi.org/10.1186/s12888-015-0640-5

Romero-Cabello, R., González-Salas, I. C. y Sánchez, C. J. (2016). Trastorno de la personalidad: un desafío en la relación médico-paciente. Atención Familiar, 23(4), 155-159. https://doi.org/10.1016/j.af.2016.10.005

Sansone, R. A. y Sansone, L. A. (2015). Borderline personality disorder in the medical setting: suggestive behaviors, syndromes, and diagnoses. Innovations in clinical neuroscience, 12(7-8), 39. Recuperado de: https://www.ncbi.nlm.nih.gov/pmc/articles/PMC4558791/pdf/icns 12 7-8 39.pdf

Semerari, A. y Dimaggio, G. (2008). Los trastornos de la personalidad. Modelos y tratamientos. Bilbao, España: Descleé de Brouwer.

Soler, J., Pascual, J. C., Tiana, T., Cebrià, A., Barrachina, J., Campins, M. J., ... y Pérez, V. (2009). Dialectical behaviour therapy skills training compared to standard group therapy in borderline personality disorder: a 3-month randomised controlled clinical trial. Behaviour research and therapy, 47(5), 353-358. https:// doi.org/10.1016/j.brat.2009.01.013

Turner, R. M. (2000). Naturalistic evaluation of dialectical behavior therapy-oriented treatment for borderline personality disorder. Cognitive and Behavioral Practice, 7(4), 413-419. https://bit.ly/2XrLjr9

Verheul, R., Van Den Bosch, L. M., Koeter, M. W., De Ridder, M. A., Stijnen, T. y Van Den Brink, W. (2003). Dialectical behaviour therapy for women with borderline personality disorder. The British Journal of Psychiatry, 182(2), 135-140. https://doi.org/10.1192/bjp.182.2.135

Zanarini, M. C., Frankenburg, F. R., Hennen, J., Reich, D. B. y Silk, K. R. (2005). The McLean Study of Adult Development (MSAD): overview and implications of the first six years of prospective follow-up. Journal of personality disorders, 19(5), 505-523. https://doi.org/10.1521/pedi.2005.19.5.505

Zanarini, M. y Jager-Hyman, S. (2009). Dissociation in Borderline Personality Disorder. En, P. Dell y J. O’Neil, Dissociation and the Dissociative Disorders. DSM-V and Beyond (pp 487-494). Nueva York, NY: Taylor \& Francis. 